\title{
Heliospheric modulation and periodicities of galactic cosmic rays during 21-24 solar cycles
}

\author{
Partha Chowdhury 1 \\ School of Space Research, Kyung Hee University, Yongin-Si, Gyeonggi-do, Republic of Korea \\ E-mail: parthares@gmail.com
}

\section{K.Kudela}

Institute of Experimental Physics, Slovak Academy of Sciences, Košice, Slovakia

E-mail: kkudela@saske.sk

\section{Y.-J.Moon}

School of Space Research, Kyung Hee University, Yongin-Si, Gyeonggi-do, Republic of Korea

E-mail: moonyjekhu.ac.kr

\begin{abstract}
Galactic cosmic rays (GCRs) encounter an outward-moving solar wind with cyclic magnetic-field fluctuation and turbulence. This causes convection and diffusion in the heliosphere. The GCRs counts from the ground-based neutron monitor (NM) stations show intensity changes that are anti-correlated with the sunspot numbers with a lag of a few months. In this paper, we make a detailed correlative study between GCRs intensity at mid cut-off rigidity station (Lomnicky stit NM) and different solar/interplanetary parameters for cycles 21-24. We find a clear asymmetry in the cross-correlation between GCRs and solar/heliospheric activity indicators for both odd and even-numbered solar cycles. The time-lags between GCRs and solar/heliospheric parameters are found different in different solar cycles as well as in the opposite polarity states $(\mathrm{A}<0$ and $\mathrm{A}>$ 0 ) within the same solar cycle. Further, we have studied the mid-term periodicities of GCRs and found the presence of well - known Rieger type and quasi-biennial oscillations (QBOs) in the power spectrum. Possible explanations of the observed results are discussed in light of $3 \mathrm{D}$ modulation models, including drift effect.
\end{abstract}

The 34th International Cosmic Ray Conference

30 July- 6 August, 2015

The Hague, The Netherland

\section{${ }^{1}$ Speaker}




\section{Introduction}

Many studies have shown that the intensity and energy spectra of GCRs undergo heliospheric modulation under the influence of solar output and its variation. GCRs arrive at the Earth with intensity modulated by the $\sim 11$-year sunspot cycle with the opposite phase and with some time lag. GCRs intensity curve also appears to follow a 22-year cycle with alternate maxima being flat topped and peaked. The drift/diffusive propagation of GCRs through the heliospheric disturbances is believed to cause the time lag [1]. The amplitude of GCRs modulation varies during different solar cycles depending on the strength of the solar magnetic field [2]. The details of the GCRs modulation and variation of time-lag factor are still a matter of interest aiming to assess the continuously changing behavior of the solar magnetic field and its influence on the propagation of GCRs during odd/even solar cycles. Like the solar parameters, GCRs also exhibit different short and mid-term periodicities [3-8]. We have investigated the correlation between GCRs intensity and various solar/heliospheric/geomagnetic activity parameters (SSN, $10.7 \mathrm{~cm}$. solar flux, IMF and Ap) considering time-lag and periodicities for mid cut-off rigidity NM situated at Lomnicky stit (LS, $3.84 \mathrm{GV}$, lat $=49.20^{\circ} \mathrm{N}$, long $=20.22^{\circ} \mathrm{E}$, alt $=2634 \mathrm{~m}$ asl.).

\section{Data and Analysis Methods}

27-day means of LS NM data from 1982 to 2013, sunspot number (SSN), solar flux (SF 2800MHz), IMF (abs B) and geomagnetic activity index Ap are used (Fig.1). Most of the data have been taken from the site of NOAA (fttp://fttp.ngdc.noaa.gov/STP/SOLAR DATA/...html). We analyze the characteristic difference between even and odd solar cycles considering the timelag between GCRs intensity and the proxy indices of solar activity, as well as the mid - term periodicities. To study the variation and the time-lag between GCRs and various solar activity indices, 'the running cross-correlation coefficients' have been calculated. We have used a timewindow of width $\mathrm{T}$ centered at time $t$, i.e., $\left[t-\frac{T}{2}, t+\frac{T}{2}\right]$. Time window of width $\mathrm{T}=50(1350$ days) was used. The cross-correlation coefficient $\mathrm{C}(\mathrm{t})$ is calculated for each time $\mathrm{t}$ using a timewindow shift (lag) in the range from -20 to +20 ( \pm 540 days). Then the time window is shifted in time by a small time step $\Delta \mathrm{t}<\mathrm{T}$, where $\Delta \mathrm{t}=27$ days and the new cross-correlation matrix is calculated. No initial time shift between the two series is used in calculating the cross-correlation matrix. Thus, we obtain a two-dimensional array for each of the solar activity data and time window. For each array and time when the cross-correlation coefficient reaches its maximum, the corresponding time shift is defined as the cross-correlation lag (L) $[9,10]$. We have also investigated the time-evolution of the intermediate term quasi-periodicities $(>27$ days $\&<11$ years) using Morlet wavelet technique setting $\omega_{0}=6$ and considering a red-noise background. 


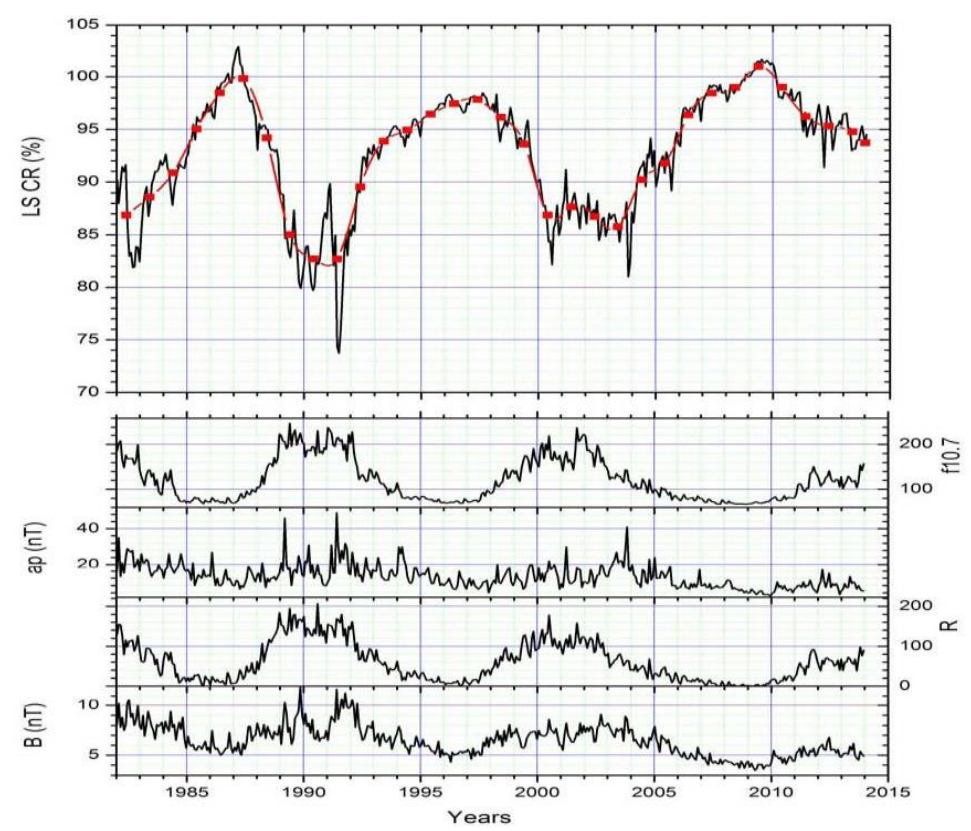

Figure1. Plot of GCRs and other solar/heliospheric/geomagnetic parameters for 1982- 2013.

The GCRs time series has a strong periodicity of $\sim 11$ years. This 'solar cycle periodicity' reduces the confidence levels of the intermediate periodicities. Therefore to dig out significant intermediate periodicities in any time profile $f\left(t_{i}\right)$, we have used the following method.

First, time profile of the low-frequency (long-period) component $f^{\mathrm{sm}}\left(t_{i}\right)$ is found by smoothing the original signal time profiles $f\left(t_{i}\right)$ using a moving average method with a suitable interval $\tau$. After smoothing, the high-frequency (short-period) component is picked out by subtracting the low-frequency component from the original signal obtaining:

$$
f^{\mathrm{hf}}{ }_{\tau}\left(\mathrm{t}_{\mathrm{i}}\right)=f\left(t_{i}\right)-f^{\mathrm{sm}}{ }_{\tau}\left(\mathrm{t}_{\mathrm{i}}\right) .
$$

Intermediate periodicities in the resulting time-series $f^{\mathrm{hf}}{ }_{\tau}\left(t_{i}\right)$ are investigated by using Wavelet techniques. To avoid the appearance of false periodicities due to filtering, we have repeated the procedure for a broad range of smoothing intervals $\tau$ from 2 to 60 months. We have used smoothing intervals 2-10 months for studying the presence and temporal variation of short and Rieger type periodicities: (i) interval of 30 months for studying quasi-biennial periodicities especially $1.2-2.5$ years and (ii) 60 months interval for studying other QBPs as well as investigating long term periodicities like 5 - 6 years as well as solar cycle variations.

\section{Results}

Correlation between 27 day average count rates of GCRs intensity and solar/ interplanetary/ geomagnetic indices is illustrated in Fig 2. The time-lag between GCRs \& other parameters for different solar cycles is apparent from the figure. The lines in the upper part of each panel show the cross-correlation coefficient (CC) and the lower panels give the cross-correlation lag (L) between GCRs and solar parameters. 

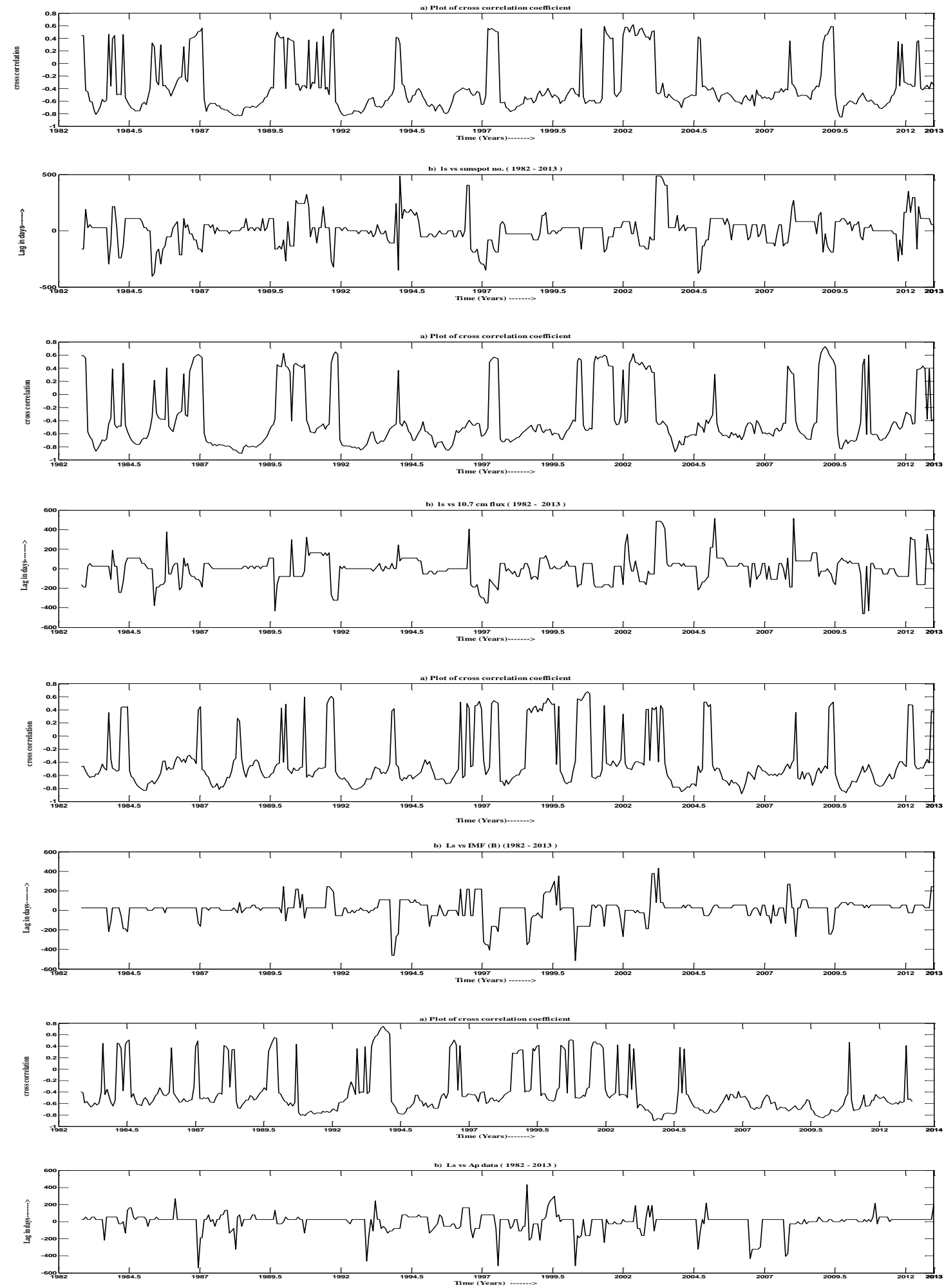

Fig 2. Running cross-correlation coefficient and time lag between the data of GCRs and different solar parameters for the period of 1982 - 2013. 
Table 1 represents the values of time lag between GCRs and different solar parameters during different solar cycles under study. We have studied the time lag in different solar cycles as well as different polarity states of the heliosphere. Corresponding results are displayed in Table 2. A $>0$ state is defined as the times when the polarity of solar magnetic field is outward in the northern hemisphere and inward in the south, such as $\sim 1991-2000$. During $\mathrm{A}<0$ states such as $\sim 1981-1990$ and 2001-2013, the direction of solar magnetic field is reversed.

Table 1: Maximum correlation coefficient and time lag between GCRs and other parameters during different solar cycles (21 -24)

\begin{tabular}{|c|c|c|c|c|}
\hline & \multicolumn{4}{|c|}{ Time-lag (L) days } \\
\hline \multirow{2}{*}{$\begin{array}{c}\text { Solar } \\
\text { cycle }\end{array}$} & SSN & 10.7 & IMF ( B) & Ap \\
\hline $21 \mathrm{D}$ & 216 & 378 & 27 & 270 \\
& 0.812 & -0.867 & -0.828 & -0.647 \\
\hline 22 & 486 & 324 & 243 & 243 \\
& -0.835 & -0.898 & -0.815 & -0.810 \\
\hline 23 & 486 & 513 & 432 & 432 \\
& -0.769 & -0.876 & -0.88 & -0.898 \\
\hline $24 \mathrm{R}$ & 351 & 351 & 243 & 216 \\
& -0.858 & -0.837 & -0.839 & -0.846 \\
\hline
\end{tabular}

Table 2: Maximum correlation coefficient and time lag between GCRs and other parameters during different solar polarity states

\begin{tabular}{|c|c|c|c|c|}
\hline & \multicolumn{4}{|c|}{ Time-lag (L) days } \\
\hline $\begin{array}{c}\text { Solar } \\
\text { polarity }\end{array}$ & SSN & 10.7 & IMF ( B) & Ap \\
\hline A $<0$ & 270 & 378 & 81 & 270 \\
$1981-1990$ & -0.833 & -0.901 & -0.833 & -0.677 \\
\hline A $>0$ & 486 & 405 & 351 & 432 \\
$1991-2000$ & -0.835 & -0.846 & -0.815 & -0.788 \\
& & & & \\
& & & & 216 \\
A $<0$ & 486 & 513 & 432 & -0.898 \\
$2001-2013$ & -0.843 & -0.876 & -0.88 & \\
& & & & \\
\hline
\end{tabular}

One can see that time-lag between GCRs and other parameters larger in odd numbered cycles comparing with even numbered cycles except with SSN which is different from previous results.

Figure 3(a-c) represents the power spectrum of GCRs time-series recorded by LS station for the period (1982 -2013) by Morlet wavelet method. The well-known Rieger type periods (130 
- 180 days) are prominent in all the solar cycles under study. In cycle 21, these groups of periods were prominent during 1983 - 1984; about 1989 to about 1992 in cycle 22; around 2000, around 2004 and 2006 during cycle 23 and in cycle 24 it persisted around 2012. The quasi-biennial oscillations (QBO) (1.2- 2.5 years) were significant from 1985 - 1994 covering the descending phase of cycle 21 and a major part of cycle 22. During cycle 23, QBO was prominent between 1999- 2005 .Power spectral analysis also exhibited a significant contour of period $\sim 5$ years between 1987 - 1994, concentrated mainly in cycle 22. Along with it, we have detected a prominent solar cycle variation period of $\sim 11$ years.
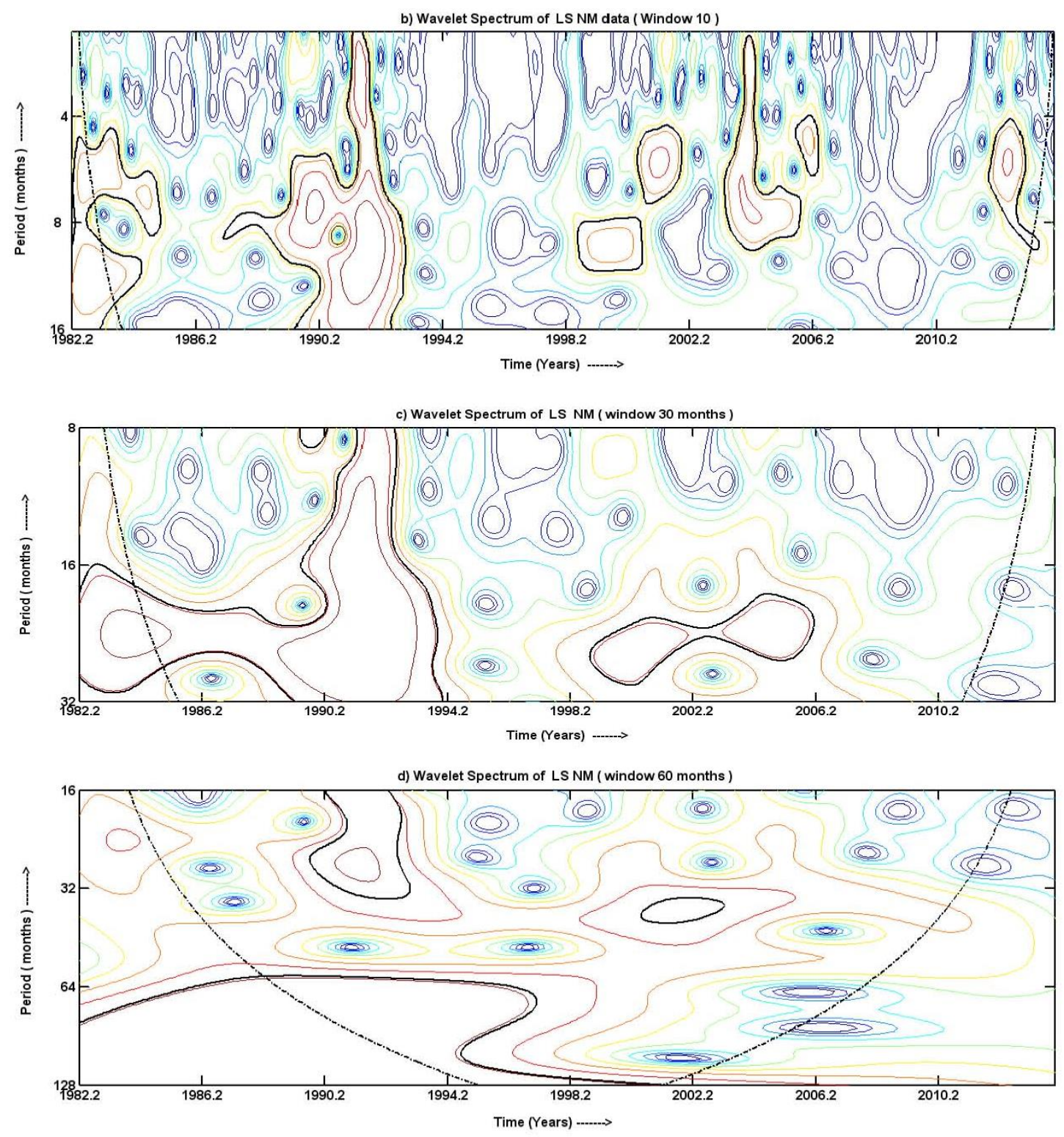

Fig 3. Morlet wavelet picture of GCRs time series for 1982 -2013. Thick black contours represents $>95 \%$ confidence level. The dot line indicate "cone of influence". 


\section{Discussion and Conclusions}

In the present work, a detailed investigation has been carried out to study the GCRs modulation, as observed at a mid-latitude high altitude NM, with respect to different solar indices and the mid-term periodicities for the solar cycles 21 -24. It is evident from Table $1 \mathrm{http} / /$ www.sciencedirect.com/science/article/pii/S1384107611000248 - t0010, that time-lag of GCRs is different during odd and even numbered solar cycles. From the cross-correlation and time-lag plots, it appears that the changes/modulation nature of GCRs with solar activity are different in even and odd solar cycles. The present analysis also shows that during some epochs of all solar cycles (21-24), GCRs are in phase with solar indices, i.e. there exists zero time-lag. This zero time-lag becomes more prominent between the time series of IMF (B); Ap and GCRs. Even during some epochs of all solar cycles, the lag is found to be negative. A negative time-lag implies that the recovery of GCRs intensity during those epochs of the cycle is faster than solar activity and probably due to unusual reversal of the global solar magnetic field, leading to an unusual heliospheric structure [11, 12].

Our power spectral analysis method exhibits the presence of Rieger type periods and QBOs. The QBOs (recently reviewed e.g. in [13]) are highly irregular resembling a set of intermittent pulses/waves with signatures of stochasticity with amplitude that varies with time. Most probably, QBOs are responsible for the Gnevyshev Gap phenomenon and the step-like decreases typical for the GCRs modulation. It is assumed that 22 year modulation of GCRs depends on time-dependent heliospheric drifts and outward propagating diffusive barriers. The propagative diffusive barriers are formed by the merging of CMEs, shocks and high-speed flows of solar wind from the Sun (Merged Interaction Regions, MIRs) as well as by "Global" MIR (GMIR), the interaction regions which extend $360^{\circ}$ around the Sun in the ecliptic plane and close over the poles. GMIRs are manifested by magnetic field enhancements, responsible for the steplike changes in the GCRs intensity around solar maxima [14]. However, the role of GMIRs regarding the $\sim 11$ year GCRs modulation cycle is still debated [15].

The GCRs modulation inside the heliosphere is a complex phenomenon which occurs all over the heliosphere and depends on many factors. No single solar index, however sophisticated, can account for it. The exact detailed physical description for the lag variation is not simple and requires further study. For a possible explanation of the lag during odd/even cycles, a variable GCRs recovery time, dependent on the polarity of the global solar magnetic field and impact of the global solar magnetic field on the heliosphere is significant. More rigorous study about this topic is underway.

\section{Acknowledgement}

This work was supported by the BK21 plus program through the National Research Foundation (NRF) funded by the Ministry of Education of Korea. KK acknowledges VEGA agency project 2/0040/13 for supporting measurements by NM at LS. 


\section{References}

[1] J.R. Wang, Dynamics of the eleven-year modulation of galactic cosmic rays. Astrophys. J., 160: 261- 281, 1970.

[2] K.Nagashima \& I. Morishita, Long term modulation of cosmic rays and inferable electro state in solar modulating region. Planetary and Space Science, 28, 177-194, 1980.

[3] A.Gil \& M.V. Alania, Cycling Changes in the Amplitudes of the 27-Day Variation of the Galactic Cosmic Ray Intensity. Solar Physics, 278(2), 447-455, 2012.

[4] K.Kudela et al., The low-frequency spectral behavior of cosmic ray intensity. J. Geophys. Res. (ISSN 0148-0227), 96, 871-875, 1991.

[5] K.Kudela et al., On Mid-Term Periodicities in Cosmic Rays.Solar Physics, 266(1), 173180,2010 .

[6] M.Laurenza et al., Quasi-biennial Modulation of Galactic Cosmic Rays. Astrophys. J., 749(2), 167-178, 2012.

[7] Partha Chowdhury et al., Evaluation of the short and intermediate term periodicities in cosmic ray intensity during solar cycle 23. Planetary and Space Science, 58(7-8), 1045-1049, 2010

[ 8] C.Kato et al., 2003, A 1.7-year quasi-periodicity in cosmic ray intensity variation observed in the outer heliosphere. J. Geophys. Res., 108(A10), SSH 4-1, CiteID 1367, 2003.

[9] I.G. Usoskin et al., Correlative study of solar activity and cosmic ray intensity.1998, Journal of Geophysical Research, 103(A5), 9567-9574, 1998.

[10] M.Minarovjech \& and K.Kudela, Cosmic Rays and Solar Coronal Emissions: CrossCorrelations of the Time Series. Solar Physics, 224(1-2), 285-290, 2004.

[11] I.G. Usoskin et al., Dependence of cosmic rays on solar activity for odd and even solar cycles. Advances in Space Research, 27(3), 571-576, 2001.

[12] Partha Chowdhury et al., Heliospheric Modulation of Galactic Cosmic Rays During Solar Cycle 23.Solar Physics, 286(2),577-591, 2013.

[13] G.Bazilevskaya et al., A Combined Analysis of the Observational Aspects of the Quasibiennial Oscillation in Solar Magnetic Activity. Space Sci Rev., 186, 359-386, 2014.

[14] L.F.Burlaga et al., Cosmic ray modulation and the distant heliospheric magnetic field Voyager 1 and 2 observations from 1986 to 1989. Journal of Geophysical Research, 98(A1), 1-11, 1993.

[15] M.S.Potgieter, Solar cycle variations and cosmic rays. Journal of Atmospheric and SolarTerrestrial Physics, 70(2-4), 207-218,2008. 